\title{
Laparoscopic Surgery for Diverticulum of the Abdominal Esophagus: A Case Report
}

\author{
Naoko Iwahashi Kondo ${ }^{1 *}$, Kazuoki Hizawa ${ }^{2}$, Dai Kitagawa ${ }^{1}$, Eisuke Adachi ${ }^{1}$, Yoichi Ikeda ${ }^{1}$ \\ ${ }^{1}$ Department of Surgery, Kyushu Central Hospital, Fukuoka, Japan \\ ${ }^{2}$ Department of Gastroenterology, Kyushu Central Hospital, Fukuoka, Japan
}

Received: March 15, 2016; Accepted: April 21, 2016; Published: June 01 , 2016

*Corresponding author: Naoko Iwahashi Kondo, Department of Surgery, Kyushu Central Hospital, 3-23-1 Shiobaru, Minami-ku, Fukuoka, 8158588 Japan, Tel: +81-92-541-4936; Fax: +81-92-541-4540; E-mail: kondo-n@surg2.med.kyushu-u.ac.jp

\begin{abstract}
Diverticulum of the abdominal esophagus is an extremely rare disease. We present a patient with diverticulum of the abdominal esophagus who could be treated successfully by a laparoscopic diverticulectomy with supporting intra-operative endoscopy. In patient with epiphrenic diverticulum, diverticulectomy with myotomy and partial fundoplication is recommended as surgical treatment. This procedure has been excogitated to control of symptoms due to esophageal motility disorders and to prevent postoperative symptoms due to reflux, stenosis, and leak. Esophageal motility disorders are regarded as the underlying cause of the epiphrenic diverticulum. Considering optimal treatment for the patients with esophageal diverticulum, it should be elucidated the relation between esophageal diverticulum and postoperative symptoms. Here we describe the first case of a laparoscopic diverticulectomy in patient with diverticulum of the abdominal esophagus, and discuss about surgical treatment of esophageal diverticulum.
\end{abstract}

Keywords: Laparoscopic surgery; Esophageal diverticula; Abdominal esophagus

\section{Introduction}

Most pulsion diverticula of the distal esophagus occur above the diaphragm, which is named as epiphrenic diverticula of the esophagus. Few cases of diverticula, arising from the abdominal esophagus below the diaphragm, have been reported. Because of the fact that epiphrenic diverticula of the esophagus are often concomitant with an esophageal motility disorder, such as achalasia, diffuse esophageal spasm, or non-specific esophageal motility disorder, a motility disorder is regarded as the underlying cause of the epiphrenic diverticulum [1] and it has been advocated that optimal surgical treatment for epiphrenic diverticula of the esophagus is diverticulectomy with myotomy and partial fundoplication [1-3]. However, it is unclear that this procedure is proper as surgical treatment for diverticulum of the abdominal esophagus, because it is unknown whether pathophysiological features of diverticula of the abdominal esophagus are same as those of epiphrenic diverticula.

We describe a case of a female with a diverticulum of the abdominal esophagus who presented with upper abdominal pain and without any findings of motility disorders on preoperative endoscopic and radiological examinations. Laparoscopic diverticulectomy with supporting intra-operative endoscopy produced satisfactory outcome in the patient. We also review and discuss about surgical treatment for diverticula of the abdominal esophagus, comparing to epiphrenic esophageal diverticula.

\section{Case Presentation}

A 50-year-old female visited to a clinic complaining of postprandial upper abdominal pain of 5 years' duration. The symptom had become serious gradually. Gastrointestinal endoscopy in the clinic revealed diverticulum of the lower part of the esophagus and food residue in the diverticulum (Figure 1). She was referred to our hospital for further examinations and treatment. A barium swallow study showed a subphrenic saccular protrusion arising from the abdominal esophagus and no abnormal motility findings. The diameter of the diverticular neck was measured $1.2 \mathrm{~cm}$ (Figure 2). Computed tomography (CT) revealed that diverticulum of $6.5 \mathrm{~cm}$ in diameter was located in the abdominal cavity and on the right side of the stomach (Figure 3).

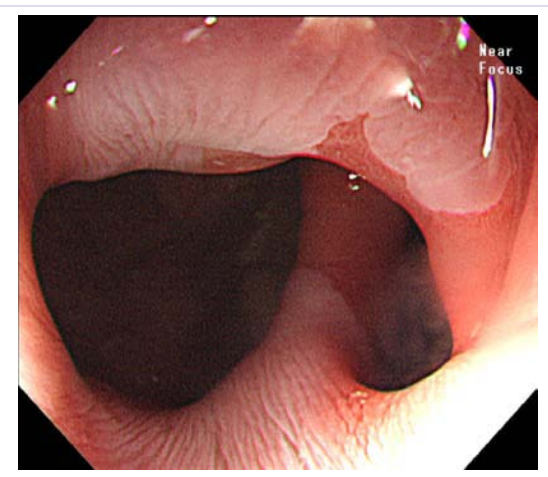

Figure 1: Preoperative gastrointestinal endoscopy. An orifice on the left side is the cardia of the stomach and the other on the right side is an entrance of diverticular pouch of the esophagus. Food residue in the diverticulum was depicted. 


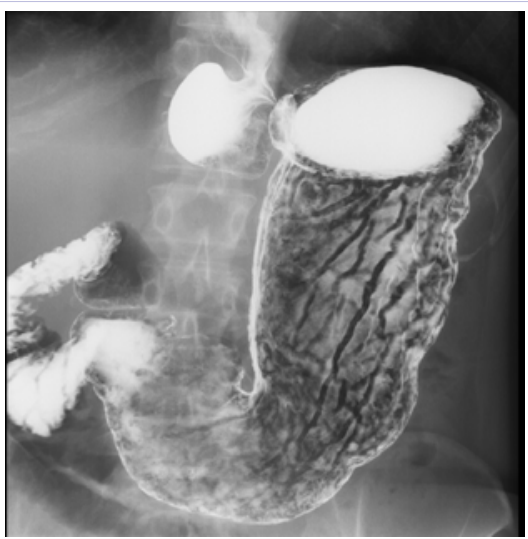

Figure 2: Preoperative barium swallow study. Barium flew into a subphrenic diverticulum of the abdominal esophagus. No abnormal esophageal motility findings were observed while the study.

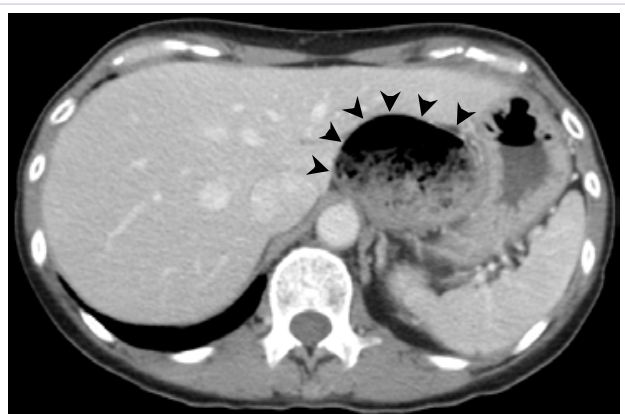

Figure 3: Computed tomography (CT) of the abdomen and the thorax. A diverticulum was located in the abdominal cavity but not in the thoracic cavity (arrowheads).

Laparoscopic diverticulectomy was performed with using an intra-operative gastrointestinal endoscope. The patient was positioned in supine position with legs apart with $30^{\circ}$ reverse Trendelenburg. Pneumoperitoneum was established and five trocars (two $12 \mathrm{~mm}$ and three $5 \mathrm{~mm}$ ) were inserted as shown in Figure 4. The diverticular pouch was located beneath the lesser omentum and adhered to the adjacent tissues (Figure 5a). The diverticular pouch was dissected until its neck became to be freed (Figure 5b). Then, an intra-operative endoscope was inserted into the esophagus. After aspiration of food residue from the diverticular pouch, the endoscope was advanced into the stomach. The diverticular neck was resected using an ECHELON FLEX ${ }^{\mathrm{TM}}$ Powered ENDOPATH ${ }^{\circledR}$ Stapler with a $60-\mathrm{mm}$ white cartridge that is intended for use in vascular/thin tissue (Ethicon Endo-Surgery, LLC, Guaynabo, PR). After the stapler jaw was closed, an endoscope was withdrawn to avoid that too much esophagus was resected and to observe the resection line from inside of the esophagus (Figure $5 \mathrm{c}$ ). The stapler was then fired and its closure was verified (Figure 5d). Nasogastric tube was put into the stomach. Drain was placed below the left lobe of the liver. The operative time was 180 minutes. Histopathologic examination of the specimen showed an inflamed diverticulum of the esophagus without any malignant findings.
A nasogastric tube was left in place until postoperative day 5 and removed after a contrast medium injection study demonstrates no leaks and smooth passage from the esophagus to the stomach (Figure 6). Food intake was resumed on the 7th postoperative day. She could eat without any symptoms. The postoperative course was uneventful and she discharged from the hospital on the 11th postoperative day. At a follow-up of 3 months, endoscopy revealed that there were no findings of stenosis, reflux, or residual pouch of diverticulum (Figure 7).

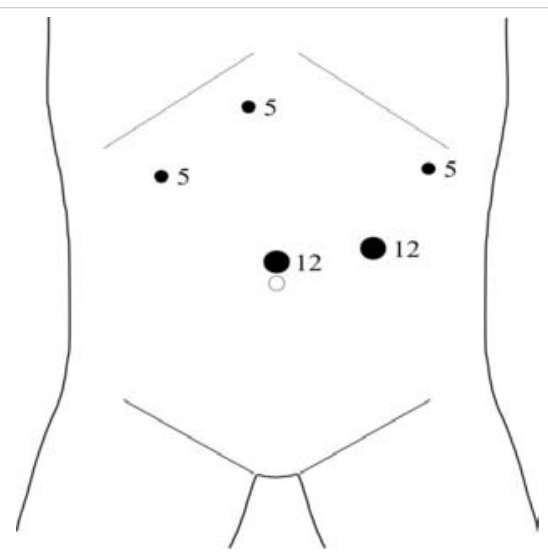

Figure 4: A schema of trocars placement. Trocar sites were shown as black dots. The number means the size of each trocar.

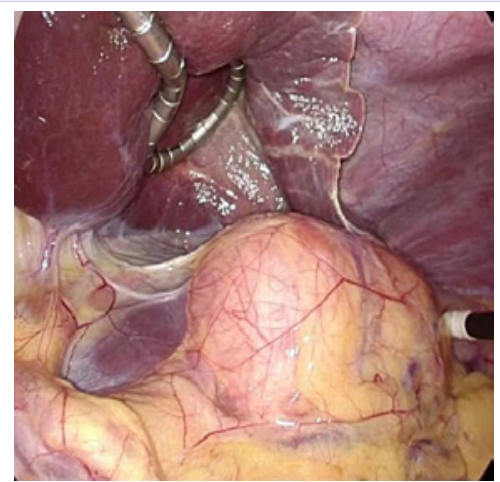

Figure 5a: Intraoperative findings. The diverticular pouch was observed beneath the lesser omentum.

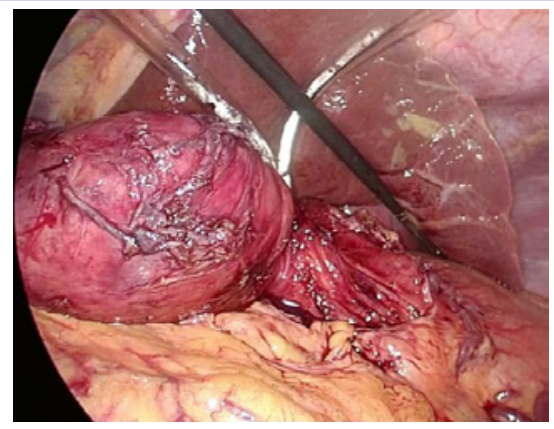

Figure 5b: Adhered tissues were dissected from the diverticulum and the diverticular neck became to be freed. 


\section{Discussion}

Esophageal motility disorders usually are concomitant with epiphrenic diverticulum, arising from lower part of the esophagus. To evaluate motility disorders, barium swallow study, esophageal manometry and/or ambulatory $\mathrm{pH}$ monitoring

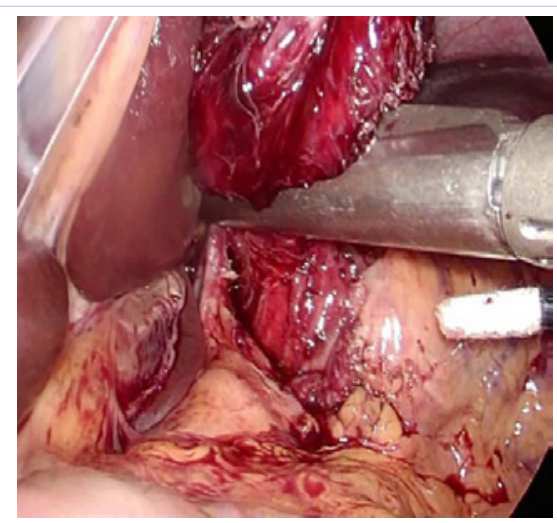

Figure 5c: After closing the jaw of a stapler, the resection line from inside of the esophagus was observed to avoid postoperative stenosis by using an intra-operative endoscope.

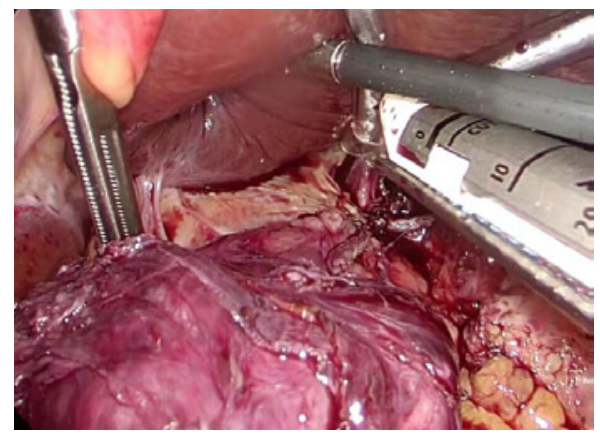

Figure 5d: Diverticulectomy was achieved. The stapler stump on the diverticular side was shown between arrows.

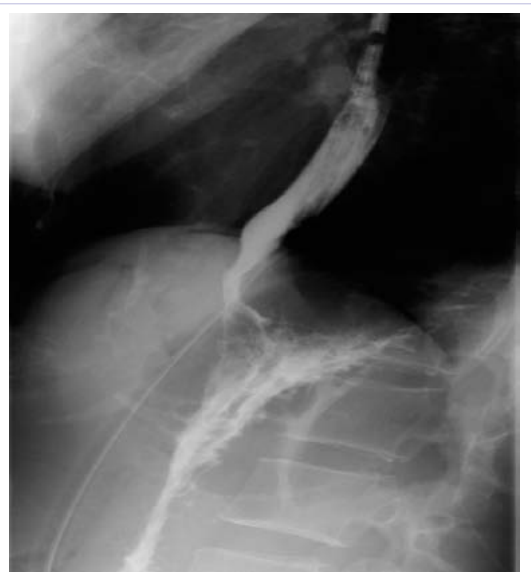

Figure 6: Postoperative contrast medium injection study. It revealed no leak of the diverticular stump and smooth passage through the esophagus.

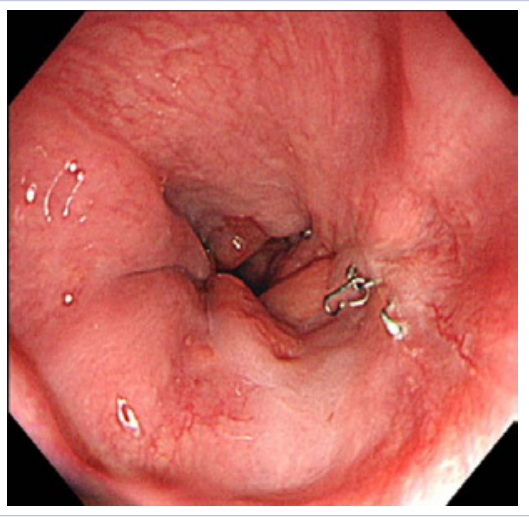

Figure 7: Postoperative endoscopy. There were no residual diverticulum and stenosis.

test are useful. Motility disorders were reported to be found in $43 \%$ of patients with epiphrenic diverticulum by barium swallow study, in $81 \%$ by using esophageal manometry [1] and in $100 \%$ by using 24-h motility recording [4]. However, relation between esophageal motility disorder and epiphrenic diverticulum remains still unclear because the motility disorders associated with an esophageal diverticulum are not always recognized and diagnosed [5]. Many studies showed that fundoplication was useful to suppress postoperative reflux, which evaluation was done by using esophageal manometry or other modalities. Matsumoto $\mathrm{H}$ et al., however, described a patient of ephiphrenic diverticulum who presented with dysphagia again 43 months after a simple diverticulectomy but her dysphagia improved 1 month later without an additional fundoplication [6]. The fact may inconsistent with that fundoplication is recommended to suppress symptoms due to postoperative reflux. Further studies of relation between motility disorder and postoperative symptoms would be required. As for diverticulum of the abdominal esophagus, we searched in database of PubMed and found only one case report of subphrenic diverticulum that esophageal motility was assessed in detail. In this report, cine-manometry was used to evaluate esophageal motility and it revealed that there was an incomplete relaxation with deglutition of the distal $2 \mathrm{~cm}$ of diverticulum neck [7]. Esophageal manometry or 24-h motility recording were not available in our institute, we assessed esophageal motility by endoscopy and barium swallow study in this case.

To prevent postoperative stenosis of the esophagus, myotomy on the opposite side of diverticulectomy is recommended in the patients with ephiphrenic diverticulum. It is also recommended that partial fundoplication with covering the site of a myotomy is added to prevent a secondary pseudodiverticulum at an uncovered myotomy [3]. To prevent a leak from the stapler stump, fundoplication with covering the diverticular stump in addition to closing the muscular wall over the stapler stump is thought to be helpful [3]. Improvement of automatic suture devices and staplers may allow decreasing leak rate without closing the muscular wall over the stapler stump. Hand sutures of the muscular wall over the stapler stump have a possibility to make the esophageal lumen narrow. A simple diverticulectomy 
by using current automatic suture device with absent of hand sutures over the stump and supporting intra-operative endoscopy should be consider as a minimum invasive and safe procedure, even concerning to prevent postoperative stenosis and leak.

There have been reported 3 surgical cases of diverticulum of the abdominal esophagus. One case was that the patient with hiatal hernia underwent diverticulectomy in thoracotomy [8]. Other case was that the patient with leiomyoma in the esophageal diverticulum underwent diverticulectomy in laparotomy [9]. The other one was that the patient underwent cardiectomy with esophagogastrostomy to avoid postoperative stenosis and reflux [10]. To our knowledge, this article was the first case report of laparoscopic approach.

Most studies have suggested that patients with absent or mildly symptomatic ephiphrenic diverticula should be managed conservatively as progression of symptoms is unlikely [5]. When to operate of symptomatic ephiphrenic diverticulum is also still unclear. In surgical cases of diverticulectomy with myotomy and partial fundoplication, average symptomatic periods are relative long that is reported 60 months [1]. Most surgeons might be too cautious in selection of surgical cases because the surgical procedures are complicated. If the primary aim of surgery in patients with diverticulum of the abdominal esophagus is to vanished serious symptoms, and if laparoscopic simple diverticulectomy allows the patients rapid relief, more patients could benefit from surgical treatments. This case was indicated a possibility that laparoscopic simple diverticulectomy is the minimum invasive surgical treatment for patients with diverticulum of the abdominal esophagus.

\section{References}

1. Tedesco P, Fisichella PM, Way LW, Patti MG. Cause and treatment of epiphrenic diverticula. Am J Surg. 2005;190(6): 902-905. doi: 10.1016/j.amjsurg.2005.08.016.

2. Rosati R, Fumagalli U, Bona S, Bonavina L, Peracchia A. Diverticulectomy, myotomy, and fundoplication through laparoscopy: a new option to treat epiphrenic esophageal diverticula? Ann Surg. 1998;227(2):174-178.

3. Hirano Y, Takeuchi H, Oyama T, Saikawa Y, Niihara M, Sako H, et al. Minimally invasive surgery for esophageal epiphrenic diverticulum: the results of 133 patients in 25 published series and our experience. Surg Today. 2013;43(1): 1-7. DOI 10.1007/s00595-012-0386-3.

4. Nehra D, Lord RV, DeMeester TR, Theisen J, Peters JH, Crookes PF, et al. Physiologic basis for the treatment of epiphrenic diverticulum. Ann Surg. 2002;235(3):346-354.

5. Zaninotto G, Portale G, Constantini M, Merigliano S, Guirroli E, Rissetto $\mathrm{C}$, et al. Long-term outcome of operated and unoperated epiphrenic diverticula. J Gastrointest Surg. 2008;12:1485-1490. DOI 10.1007/ s11605-008-0570-3.

6. Matsumoto H, Kubota H, Higashida M, Manabe N, Haruma K, Hirai T. Esophageal epiphrenic diverticulum associated with diffuse esophageal spasm. Int J Surg Case Rep. 2015;13:79-83. doi: 10.1016/j. ijscr.2015.06.018.

7. Coburn WM Jr, Dana ER, Gayler BW. Subphrenic esophageal diverticulum: a case studied by cine-manometry. Johns Hopkins Med J. 1971;128(1):41-44.

8. Retting J. Diverticulum of the abdominal portion of the esophagus. Gastroenterology. 1962;42(6):781-783. DOI: doi.org/10.1016/ S0016-5085(62)80128-0.

9. Wallner B, Friedrich JM, Kunz R. Leiomyoma of the esophagus in a subphrenic diverticulum. Rofo. 1988;148(6):717-718.

10.Sam AD Jr, Chaer RA, Cintron J, Teresi M, Massad MG. Upper gastrointestinal bleeding caused by a "hypophrenic" diverticulum of the distal esophagus. Am Surg. 2005;71(4):333-335. 